\title{
POISSON-GAUSSIAN DENOISING USING THE EXACT UNBIASED INVERSE OF THE GENERALIZED ANSCOMBE TRANSFORMATION
}

\author{
Markku Mäkitalo and Alessandro Foi \\ Department of Signal Processing, Tampere University of Technology \\ P.O. Box 553, 33101 Tampere, Finland \\ e-mail: firstname.lastname@tut.fi
}

\begin{abstract}
The characteristic errors of many digital imaging devices can be modelled as Poisson-Gaussian noise, the removal of which can be approached indirectly through variance stabilization. The generalized Anscombe transformation (GAT) is commonly used for stabilization, but rigorous studies regarding its unbiased inverse transformation have been neglected. We introduce the exact unbiased inverse of the GAT, show that it is of essential importance for ensuring accurate denoising, and demonstrate that our approach leads to state-of-the-art results. This paper generalizes our earlier work, in which we presented an exact unbiased inverse of the Anscombe transformation for the case of pure Poisson noise removal.
\end{abstract}

Index Terms - denoising, photon-limited imaging, variance stabilization, Poisson-Gaussian noise.

\section{INTRODUCTION}

The characteristic errors of many digital imaging devices can be modelled as Poisson-Gaussian noise, where the Poisson component accounts for the signal-dependent uncertainty inherent to photon accumulation, and the Gaussian component accounts for the other signal-independent noise sources, such as thermal noise.

While the problem of denoising images corrupted by PoissonGaussian noise can be approached by taking the noise statistics directly into account (e.g., [1]), it can also be approached indirectly through variance stabilization. This is a three-step process, where the noisy data is first modified by applying a nonlinear variancestabilizing transformation (VST). The resulting data can be treated as Gaussian with unitary variance, and thus, it can be denoised with any algorithm designed for the removal of Gaussian noise. Finally, the desired estimate is obtained by applying an inverse VST to the denoised data.

The generalized Anscombe transformation (GAT) [2] is commonly used for stabilizing the variance of Poisson-Gaussian noise. While this transformation is well known, its corresponding exact unbiased inverse transformation has been neglected, even though it is of essential importance for ensuring accurate denoising results, as we show in this work. We introduce the exact unbiased inverse of the GAT for Poisson-Gaussian noise, and in particular, we provide results also for finite parameter values, while previous works [2] have considered only the asymptotic case. Moreover, we demonstrate that

This work was supported by the Academy of Finland (project no. 213462, Finnish Programme for Centres of Excellence in Research 2006-2011, project no. 252547, Academy Research Fellow 2011-2016, and project no. 129118, Postdoctoral Researcher's Project 2009-2011) and by Tampere Doctoral Programme in Information Science and Engineering (TISE). our approach leads to state-of-the-art results. This paper generalizes our earlier work [3], [4], in which we presented an exact unbiased inverse of the Anscombe transformation [5] and showed its importance in obtaining accurate denoising results for the case of pure Poisson noise.

The rest of the paper is organized as follows: Section 2 presents some preliminaries about Poisson-Gaussian noise and about stabilizing its variance with the GAT. In Section 3, which is the core of our contribution, we discuss how to construct an exact unbiased inverse of the GAT and how it behaves asymptotically. Section 4 consists of experiments, and in Section 5 we discuss the obtained results.

\section{PRELIMINARIES}

\subsection{Poisson-Gaussian noise}

Let $\grave{z}_{i}, i=1, \ldots, N$, be the observed pixel values obtained through an image acquisition device. We model each $\grave{z}_{i}$ as an independent random Poisson variable $p$ with an underlying mean value $\grave{y}_{i}$, scaled by $\alpha>0$ and corrupted by additive Gaussian noise $n$ of mean $g$ and standard deviation $\grave{\sigma}$. In other words,

$$
\grave{z}_{i}=\alpha p+n,
$$

where $p \sim \mathcal{P}\left(\grave{y}_{i}\right)$ and $n \sim \mathcal{N}\left(g, \grave{\sigma}^{2}\right)$. Thus, we can define PoissonGaussian noise as

$$
\eta_{i}=\grave{z}_{i}-\grave{y}_{i}
$$

2.2. Variance stabilization with the generalized Anscombe transformation

Assuming $\grave{z}$ is distributed according to (1), we can apply the generalized Anscombe transformation [2]

$$
f(\grave{z})= \begin{cases}\frac{2}{\alpha} \sqrt{\alpha \grave{z}+\frac{3}{8} \alpha^{2}+\grave{\sigma}^{2}-\alpha g}, & \grave{z}>-\frac{3}{8} \alpha-\frac{\grave{\sigma}^{2}}{\alpha}+g \\ 0, & \grave{z} \leq-\frac{3}{8} \alpha-\frac{\grave{\sigma}^{2}}{\alpha}+g\end{cases}
$$

to $\grave{z}$ in order to (approximately) stabilize its variance to unity, i.e., $\operatorname{var}\{f(\grave{z}) \mid \grave{y}, \grave{\sigma}\} \approx 1$. Note that for the pure Poisson case (i.e., $\alpha=1, \sigma=0$, and $g=0$ ), this coincides with the traditional Anscombe transformation [5] used for stabilizing data corrupted by Poisson noise.

The number of parameters which define the transformation (3) can be reduced significantly by simple variable substitutions

$$
z=\frac{\grave{z}-g}{\alpha}, \quad \sigma=\frac{\grave{\sigma}}{\alpha}
$$

which affinely map $\grave{z}$ to $z$, a random (non-scaled) Poisson variable corrupted by additive Gaussian noise of mean 0 and standard devia- 
tion $\sigma$. In particular, the probability distribution of $z$ is

$$
p(z \mid y, \sigma)=\sum_{k=0}^{+\infty}\left(\frac{y^{k} e^{-y}}{k !} \times \frac{1}{\sqrt{2 \pi \sigma^{2}}} e^{-\frac{(z-k)^{2}}{2 \sigma^{2}}}\right) .
$$

Thus, according to (3), $z$ can be stabilized with the transformation

$$
f_{\sigma}(z)=\left\{\begin{array}{ll}
2 \sqrt{z+\frac{3}{8}+\sigma^{2}}, & z>-\frac{3}{8}-\sigma^{2} \\
0, & z \leq-\frac{3}{8}-\sigma^{2}
\end{array} .\right.
$$

In other words, for any of the parameters $\alpha$ and $g$, we can stabilize the variance of $\dot{z}$ by means of variable substitutions (4), followed by the transformation (6). Then, after applying an inverse transformation $\mathcal{I}$ of (6) to the denoised data $D$, we simply return to the original range by inverting (4), i.e., setting $\hat{y}=\alpha \mathcal{I}(D)+g$. Note that (4) and its inverse are affine, so they do not introduce any bias in the estimation. Thus, in the rest of the paper, we consider only the stabilization of $z$, which is the observed data after the variable substitution (4), and by GAT we refer to the corresponding transformation (6).

Figure 1(a) shows the forward transformation (6) for the parameter values $\sigma=0.01,1,2,3$, and the corresponding standard deviations of the stabilized variables $f_{\sigma}(z)$ are shown in Figure 1(b). Note that there is a particular overshoot in the standard deviation at around $\sigma=2$ for low values of $y$, but it begins to settle down towards the desired value 1 as $\sigma$ increases.

\section{EXACT UNBIASED INVERSE TRANSFORMATION}

\subsection{Definition}

Assuming the denoising of $f_{\sigma}(z)$ was successful, we may treat the denoised data $D$ as the expected value $E\left\{f_{\sigma}(z) \mid y, \sigma\right\}$. Then, the exact unbiased inverse of the generalized Anscombe transformation (6) is in fact a family of inverse transformations $\mathcal{I}_{\sigma}$, parametrized by $\sigma$, that maps the values $E\left\{f_{\sigma}(z) \mid y, \sigma\right\}$ to the desired values $E\{z \mid y, \sigma\}$ :

$$
\mathcal{I}_{\sigma}: E\left\{f_{\sigma}(z) \mid y, \sigma\right\} \longmapsto E\{z \mid y, \sigma\} .
$$

Since we trivially know $E\{z \mid y, \sigma\}=y$ for any given $y$, constructing the inverse requires us to compute the values $E\left\{f_{\sigma}(z) \mid y, \sigma\right\}$, analogously to how the exact unbiased inverse of the Anscombe transformation was computed in [3]. In this more general case, it is computed as

$$
\begin{gathered}
E\left\{f_{\sigma}(z) \mid y, \sigma\right\}=\int_{-\infty}^{+\infty} f_{\sigma}(z) p(z \mid y, \sigma) d z \\
=\int_{-\infty}^{+\infty} 2 \sqrt{z+\frac{3}{8}+\sigma^{2}} \sum_{k=0}^{+\infty}\left(\frac{y^{k} e^{-y}}{k ! \sqrt{2 \pi \sigma^{2}}} e^{-\frac{(z-k)^{2}}{2 \sigma^{2}}}\right) d z .
\end{gathered}
$$

The exact unbiased inverse transformations $\mathcal{I}_{\sigma}$ for the parameter values $\sigma=0.01,1,2,3$ are shown in Figure 1(c), along with the corresponding algebraic inverses of (6) for the comparison.

\subsection{Asymptotic behaviour}

As we explicitly construct the inverse mapping (7) only for a finite grid of values, it is also of interest to examine the asymptotic behaviour of $\mathcal{I}_{\sigma}$. When the standard deviation $\sigma$ of the Gaussian noise component is large, we may formulate $\mathcal{I}_{\sigma}$ in terms of the exact unbiased inverse Anscombe transformation $\mathcal{I}_{0}$ [3] as

$$
\mathcal{I}_{\sigma} \approx \mathcal{I}_{0}-\sigma^{2}
$$

Likewise, when $\sigma$ is very small, we may do the same approximation. For the detailed derivation of this approximation and analysis of its accuracy, we refer the reader to [6].

\subsection{Practical implementation}

Taking into account the asymptotic result (9), and given that $\mathcal{I}_{0}$ is already available (either in accurate numerical form or as closedform analytical approximation [3], [4]), to compute $\mathcal{I}_{\sigma}$ it is sufficient to tabulate $E\left\{f_{\sigma}(z) \mid y, \sigma\right\}$ (8) only for a finite grid of values, and resort to interpolation (between the grid values) and to the asymptotic form $\mathcal{I}_{0}(D)-\sigma^{2}$ (outside of the grid). In particular, for our experiments, we considered 96 non-equispaced values $\sigma \in\{0.01, \ldots, 50\}$ and 1199 non-equispaced values of $y \in$ $\{0, \ldots, 200\}$ and calculated $E\left\{f_{\sigma}(z) \mid y, \sigma\right\}$ on such $96 \times 1199$ grid. Our Matlab software implementing this inverse transformation is available online at http://www.cs.tut.fi/foi/invansc.

\section{EXPERIMENTS}

We evaluate the denoising performance associated with the proposed exact unbiased inverse $\mathcal{I}_{\sigma}$ by considering the Cameraman $(256 \times 256)$ and Fluorescent Cells $(512 \times 512)$ test images. For both images, we scale the original image to eight different peak values ( 1 , $2,5,10,20,30,60,120)$, and corrupt them with Poisson-Gaussian noise $(\sigma=$ peak/10), as was done in [1].

We denoise each image with the three-step variance stabilization approach explained in Section 1, using either BM3D [7] or BLSGSM [8] as the Gaussian denoising algorithm, and inverting the denoised data with each of the following transformations: the exact unbiased inverse $\mathcal{I}_{\sigma}$, the asymptotic inverse $\mathcal{I}_{\text {asy }}(D)=\frac{1}{4} D^{2}-\frac{1}{8}-\sigma^{2}$, or the algebraic inverse $\mathcal{I}_{\text {alg }}(D)=\frac{1}{4} D^{2}-\frac{3}{8}-\sigma^{2}$. For comparison with the direct approaches, we also denoise each image with the state-of-the-art UWT/BDCT PURE-LET method proposed in [1]. The results are presented in Table 1, where each PSNR value is an average of ten individual denoising results (performed on ten random realizations of the Poisson-Gaussian noise).

We see that the proposed method is competitive with the UWT/BDCT PURE-LET algorithm, outperforming it in many cases, in particular when variance stabilization is combined with the BM3D algorithm, which represents the state of the art in additive white Gaussian noise removal. Moreover, there are no major declines in performance for the low-intensity cases, which demonstrates the fact that the poor performance shown in earlier works (e.g., [1]) is not simply due to inadequate variance stabilization associated with the GAT, but mostly a consequence of using an improper inverse transformation. In particular, we see that for the low-intensity cases it is clearly not reasonable to use either the asymptotic inverse or the algebraic inverse; instead, the proposed exact unbiased inverse can be used everywhere.

Figures 2-3 present visual comparisons for Cameraman (peak $=80, \sigma=3$ ), corroborating the observed good performance of the proposed denoising method. Finally, Figure 4 shows the denoising results for a low-intensity Boat image (peak $=1.5, \sigma=0.1$ ), including a comparison of the different inverses. This clearly visualizes the previously noted importance of applying a proper inverse transformation to the denoised data.

\section{DISCUSSION AND CONCLUSIONS}

We have generalized our earlier work [3], [4] by proposing an exact unbiased inverse of the generalized Anscombe transformation for Poisson-Gaussian noise. We showed that the denoising performance associated with this inverse, in conjunction with a state-ofthe-art Gaussian noise removal algorithm, is competitive with that of a state-of-the-art algorithm designed specifically for the removal 


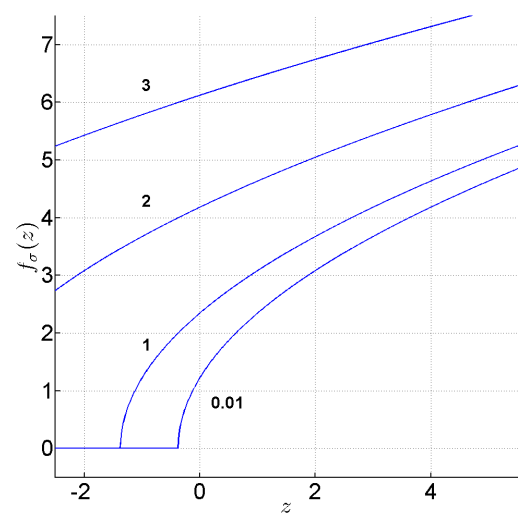

(a)

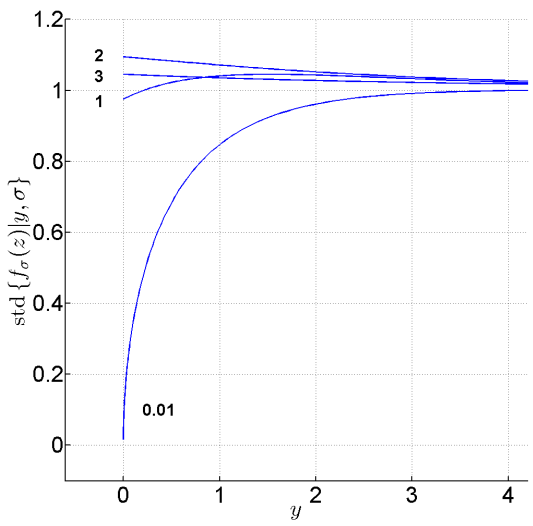

(b)

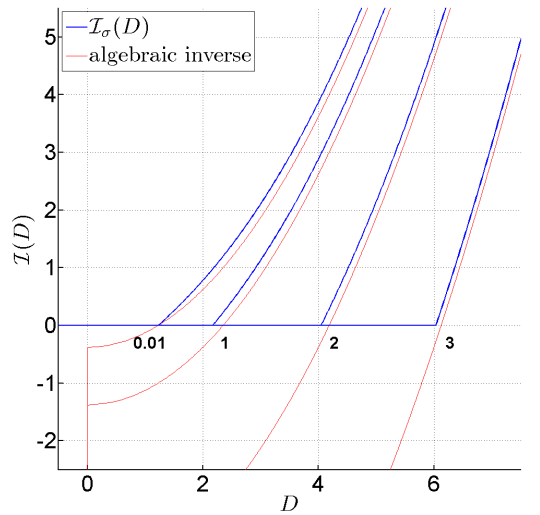

(c)

Fig. 1: The generalized Anscombe transformation (6) for the parameter values $\sigma=0.01,1,2,3$. (a) The forward transformations $f_{\sigma}(z)$, (b) The standard deviations of the stabilized variables $f_{\sigma}(z)$, (c) The exact unbiased inverse transformations $\mathcal{I}_{\sigma}$, compared with the corresponding algebraic inverses of (6).

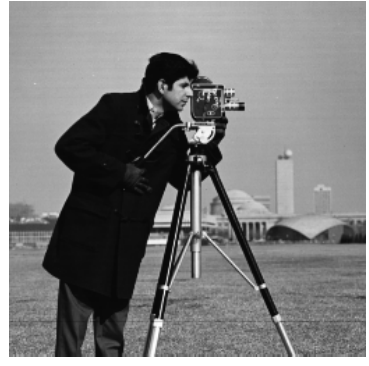

(a)

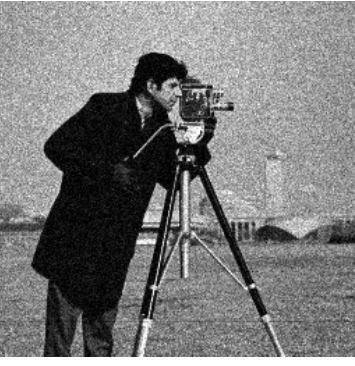

(b)

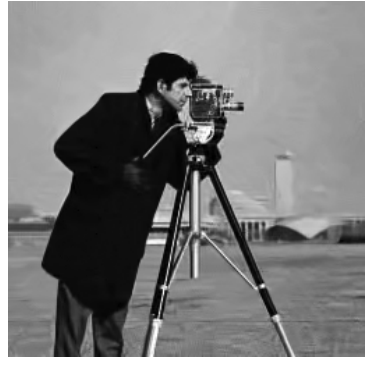

(c)

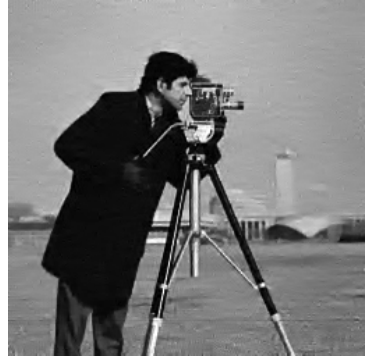

(d)

Fig. 2: The denoising of Cameraman $(256 \times 256)$. (a) Original image, (b) Noisy image (peak $=80, \sigma=3$, PSNR $=21.38 \mathrm{~dB})$, (c) Denoised with BM3D and the exact unbiased inverse $\mathcal{I}_{\sigma}(\mathrm{PSNR}=30.15 \mathrm{~dB})$, (d) Denoised with UWT/BDCT PURE-LET $(\mathrm{PSNR}=29.68 \mathrm{~dB})$.

Table 1: A comparison of the denoising performance (PSNR, dB) of several denoising algorithms and inverse transformations, using various peak intensities and various standard deviations $\sigma$ of the Gaussian noise component. The results are averages of ten independent realizations.

\begin{tabular}{|c|c|c|c|c|c|c|c|c|c|c|}
\hline \multirow[t]{2}{*}{ Image } & \multirow[t]{2}{*}{ Peak } & \multirow[t]{2}{*}{$\sigma$} & \multirow[t]{2}{*}{ Noisy } & \multicolumn{3}{|c|}{ GAT + BM3D } & \multicolumn{3}{|c|}{ GAT + BLS-GSM } & \multirow{2}{*}{$\begin{array}{c}\text { UWT/BDCT } \\
\text { PURE-LET [1] }\end{array}$} \\
\hline & & & & $\mathcal{I}_{\sigma}$ & $\mathcal{I}_{\text {asy }}$ & $\mathcal{I}_{\text {alg }}$ & $\mathcal{I}_{\sigma}$ & $\mathcal{I}_{\text {asy }}$ & $\mathcal{I}_{\text {alg }}$ & \\
\hline \multirow{8}{*}{$\begin{array}{l}\text { Cameraman } \\
(256 \times 256)\end{array}$} & $\overline{1}$ & $\overline{\overline{0.1}}$ & 3.20 & 20.23 & 15.55 & $\overline{15.72}$ & $\overline{18.46}$ & 14.56 & $\overline{15.40}$ & $\overline{20.35}$ \\
\hline & 2 & 0.2 & 6.12 & 21.93 & 20.70 & 18.24 & 20.28 & 19.41 & 17.36 & 21.60 \\
\hline & 5 & 0.5 & 9.83 & 24.09 & 24.00 & 22.36 & 23.01 & 22.93 & 21.29 & 23.33 \\
\hline & 10 & 1 & 12.45 & 25.52 & 25.52 & 24.80 & 24.36 & 24.35 & 23.62 & 24.68 \\
\hline & 20 & 2 & 14.76 & 26.77 & 26.75 & 26.48 & 25.58 & 25.53 & 25.26 & 25.92 \\
\hline & 30 & 3 & 15.91 & 27.30 & 27.29 & 27.13 & 26.20 & 26.16 & 26.01 & 26.51 \\
\hline & 60 & 6 & 17.49 & 28.07 & 28.06 & 28.01 & 27.02 & 26.98 & 26.93 & 27.35 \\
\hline & 120 & 12 & 18.57 & 28.57 & 28.55 & 28.54 & 27.57 & 27.52 & 27.51 & 27.89 \\
\hline \multirow{8}{*}{$\begin{array}{l}\text { Fluorescent Cells } \\
(512 \times 512)\end{array}$} & 1 & 0.1 & 7.22 & 24.54 & 13.86 & 20.83 & 22.35 & 13.47 & 20.01 & 25.13 \\
\hline & 2 & 0.2 & 9.99 & 25.87 & 20.99 & 21.96 & 24.20 & 20.25 & 21.23 & 26.25 \\
\hline & 5 & 0.5 & 13.37 & 27.45 & 26.93 & 24.80 & 26.99 & 26.52 & 24.25 & 27.60 \\
\hline & 10 & 1 & 15.53 & 28.63 & 28.61 & 27.20 & 28.05 & 28.03 & 26.50 & 28.59 \\
\hline & 20 & 2 & 17.21 & 29.65 & 29.64 & 29.09 & 29.05 & 28.89 & 28.28 & 29.47 \\
\hline & 30 & 3 & 17.97 & 30.16 & 30.15 & 29.86 & 29.74 & 29.65 & 29.33 & 29.84 \\
\hline & 60 & 6 & 18.86 & 30.77 & 30.77 & 30.68 & 30.52 & 30.48 & 30.38 & 30.42 \\
\hline & 120 & 12 & 19.39 & 31.14 & 31.14 & 31.11 & 30.91 & 30.87 & 30.85 & 30.70 \\
\hline
\end{tabular}




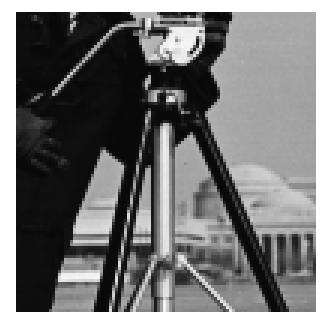

(a)

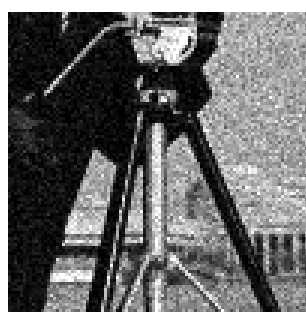

(b)

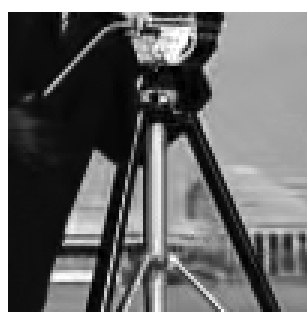

(c)

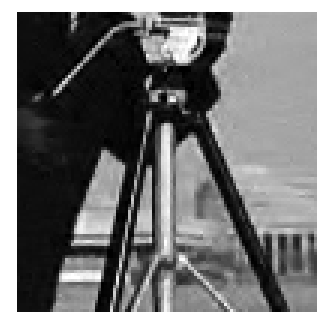

(d)

Fig. 3: A $100 \times 100$ section of each of the images in Figure 2. (a) Original image, (b) Noisy image (peak $=80, \sigma=3$ ), (c) Denoised with BM3D and the exact unbiased inverse $\mathcal{I}_{\sigma}$, (d) Denoised with UWT/BDCT PURE-LET.

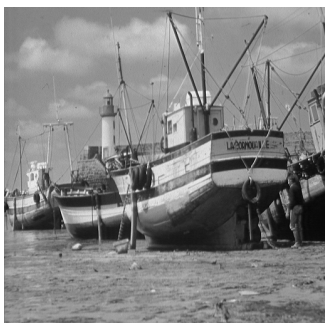

(a)

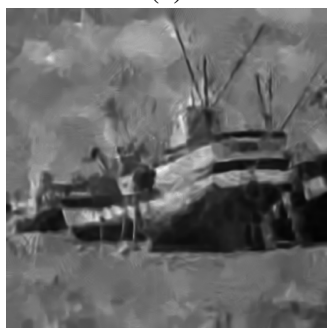

(d)

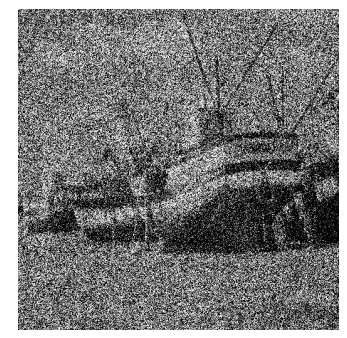

(b)

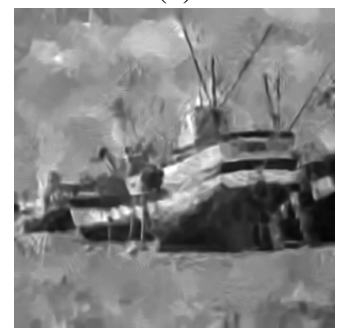

(e)

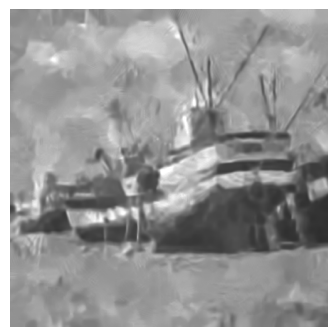

(c)

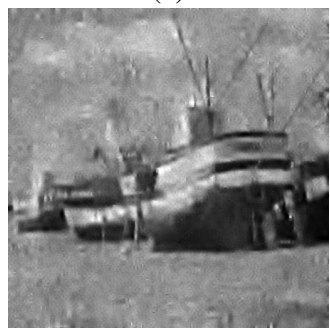

(f)

Fig. 4: The denoising of Boat $(512 \times 512)$. (a) Original image, (b) Noisy image (peak $=1.5, \sigma=0.1$, PSNR $=4.64 \mathrm{~dB}$ ), (c) Denoised with BM3D and the asymptotic inverse $\mathcal{I}_{\text {asy }}(P S N R=20.18 \mathrm{~dB}),(\mathrm{d})$ Denoised with BM3D and the algebraic inverse $\mathcal{I}_{\text {alg }}(\mathrm{PSNR}=17.34 \mathrm{~dB}),(\mathrm{e})$ Denoised with BM3D and the exact unbiased inverse $\mathcal{I}_{\sigma}(\mathrm{PSNR}=22.30 \mathrm{~dB})$, (f) Denoised with UWT/BDCT PURE-LET $(\mathrm{PSNR}=22.31 \mathrm{~dB})$.

of Poisson-Gaussian noise. Further, we observed that for low peak intensities, the performance gain obtained by using the exact unbiased inverse instead of the algebraic or the asymptotic inverse is especially significant. In other words, we showed that the poor denoising performance shown in earlier works is not simply due to the inability of the GAT to stabilize the noise variance adequately, but mostly due to applying an unsuitable inverse transformation.

For rigorous mathematical derivations and proofs concerning the results presented here, we refer the reader to our forthcoming journal paper [6], where we also show that the exact unbiased inverse is optimal in a maximum likelihood sense, and we further introduce a closed-form approximation of this inverse.

\section{ACKNOWLEGDEMENT}

We thank Florian Luisier for providing us with their PURE-LET software and the Fluorescent Cells image used in the experiments.

\section{REFERENCES}

[1] Luisier, F., T. Blu, and M. Unser, "Image Denoising in Mixed Poisson-Gaussian Noise", IEEE Trans. Image Process., vol. 20, no. 3, pp. 696-708, March 2011.

[2] Starck, J.L., F. Murtagh, and A. Bijaoui, Image Processing and Data Analysis, Cambridge University Press, Cambridge, 1998.
[3] Mäkitalo, M., and A. Foi, "Optimal inversion of the Anscombe transformation in low-count Poisson image denoising", IEEE Trans. Image Process., vol. 20, no. 1, pp. 99-109, Jan. 2011. http://dx.doi.org/10.1109/TIP.2010.2056693

[4] Mäkitalo, M., and A. Foi, "A closed-form approximation of the exact unbiased inverse of the Anscombe variance-stabilizing transformation", IEEE Trans. Image Process., vol. 20, no. 9, pp. 2697-2698, Sep. 2011. http://dx.doi.org/10.1109/TIP.2011.2121085

[5] Anscombe, F.J., "The transformation of Poisson, binomial and negative-binomial data", Biometrika, vol. 35, no. 3/4, pp. 246254, Dec. 1948. http://dx.doi.org/10.1093/biomet/35.3-4.246

[6] Mäkitalo, M., and A. Foi, "Optimal inversion of the generalized Anscombe transformation for Poisson-Gaussian noise", submitted to IEEE Trans. Image Process., Sep. 2011.

[7] Dabov, K., A. Foi, V. Katkovnik, and K. Egiazarian, "Image denoising by sparse 3D transform-domain collaborative filtering", IEEE Trans. Image Process., vol. 16, no. 8, pp. 2080-2095, Aug. 2007.

[8] Portilla, J., V. Strela, M.J. Wainwright, and E.P. Simoncelli, "Image denoising using scale mixtures of Gaussians in the wavelet domain”, IEEE Trans. Image Process., vol. 12, no. 11, pp. 1338-1351, Nov. 2003. 